\title{
Based on the Network Degeneration Hypothesis: Separating Individual Patients with Different Neurodegenerative Syndromes in a Preliminary Hybrid PET/MR Study
}

\author{
Masoud Tahmasian ${ }^{1-6}$, Junming Shao ${ }^{7-9}$, Chun Meng ${ }^{1,3}$, Timo Grimmer $^{10}$, Janine Diehl-Schmid $^{10}$, Behrooz H. Yousefi $^{2,11}$, \\ Stefan Förster ${ }^{2,3}$, Valentin Riedl ${ }^{1-3}$, Alexander Drzezga ${ }^{2,6}$, and Christian Sorg ${ }^{1-3,10}$ \\ ${ }^{I}$ Department of Neuroradiology, Technische Universität München, Munich, Germany; ${ }^{2}$ Department of Nuclear Medicine, Technische \\ Universität München, Munich, Germany; ${ }^{3}$ TUM-Neuroimaging Center (TUM-NIC), Technische Universität München, Munich, \\ Germany; ${ }^{4}$ Sleep Disorders Research Center, Kermanshah University of Medical Science (KUMS), Kermanshah, Iran; ${ }^{5}$ Department \\ of Neurology, University Hospital Cologne, Cologne, Germany; ${ }^{6}$ Department of Nuclear Medicine, University Hospital Cologne, \\ Cologne, Germany; ${ }^{7}$ School of Computer Science and Engineering, University of Electronic Science and Technology of China, \\ Chengdu, China; ${ }^{8}$ Big Data Research Center, University of Electronic Science and Technology of China, Chengdu, China; ${ }^{9}$ Center for \\ Information in BioMedicine, University of Electronic Science and Technology of China, Chengdu, China; ${ }^{10}$ Department of Psychiatry \\ and Psychotherapy, Technische Universität München, Munich, Germany; and ${ }^{11}$ Radiopharmacy of Klinikum Rechts der Isar, \\ Technische Universität München, Munich, Germany
}

The network degeneration hypothesis (NDH) of neurodegenerative syndromes suggests that pathologic brain changes distribute primarily along distinct brain networks, which are characteristic for different syndromes. Brain changes of neurodegenerative syndromes can be characterized in vivo by different imaging modalities. Our aim was to test the hypothesis whether multimodal imaging based on the $\mathrm{NDH}$ separates individual patients with different neurodegenerative syndromes. Methods: Twenty patients with Alzheimer disease (AD) and 20 patients with frontotemporal lobar degeneration (behavioral variant frontotemporal dementia [bvFTD, $n=11$ ], semantic dementia [SD, $n=4$ ], or progressive nonfluent aphasia [PNFA, $n=5]$ ]) underwent simultaneous MRI and ${ }^{18} \mathrm{~F}$-FDG PET in a hybrid PET/MR scanner. The 3 outcome measures were voxelwise values of degree centrality as a surrogate for regional functional connectivity, glucose metabolism as a surrogate for regional metabolism, and volumetric-based morphometry as a surrogate for regional gray matter volume. Outcome measures were derived from predefined core regions of 4 intrinsic networks based on the NDH, which have been demonstrated to be characteristic for AD, bvFTD, SD, and PNFA, respectively. Subsequently, we applied support vector machine to classify individual patients via combined imaging measures, and results were evaluated by leave-one-out cross-validation. Results: On the basis of multimodal voxelwise regional patterns, classification accuracies for separating patients with different neurodegenerative syndromes were $77.5 \%$ for $A D$ versus others, $82.5 \%$ for bvFTD versus others, $97.5 \%$ for SD versus others, and $87.5 \%$ for PNFA versus others. Multimodal classification results were significantly superior to unimodal approaches. Conclusion: Our finding provides initial evidence that the combination of regional metabolism, functional connectivity, and gray matter volume, which were derived from disease characteristic networks, separates individual patients with different neurodegenerative syndromes. Preliminary results suggest that employing multimodal

Received Aug. 13, 2015; revision accepted Oct. 29, 2015.

For correspondence or reprints contact: Masoud Tahmasian, Department of Neurology, University Hospital Cologne, Kerpener Strasse 62, 50937, Cologne, Germany.

E-mail: masoud.tahmasian@uk-koeln.de

Published online Nov. 19, 2015.

COPYRIGHT (c) 2016 by the Society of Nuclear Medicine and Molecular Imaging, Inc. imaging guided by the NDH may generate promising biomarkers of neurodegenerative syndromes.

Key Words: neurodegenerative syndromes; network degeneration hypothesis; Alzheimer's disease; frontotemporal lobar degeneration; hybrid PET/MR

J Nucl Med 2016; 57:410-415

DOI: 10.2967/jnumed.115.165464

B neurodegenerative syndromes result in dementia characterized by progressive cognitive and behavioral dysfunction (1). In line with clinical variation of distinct dementia syndromes (e.g., sociobehavioral and language symptoms stand out in frontotemporal lobar degeneration [FTLD], whereas memory and attention deficits dominate in Alzheimer disease $[\mathrm{AD}]$ ), cumulative evidence suggests that neurodegenerative syndromes do not distribute randomly across the brain but primarily affect specific functional networks, corresponding roughly with cognitive-behavioral functions (2-5). The network degeneration hypothesis $(\mathrm{NDH})$ of neurodegenerative syndromes suggests that both initiation and propagation of pathologic changes are happening primarily along specific brain networks $(4,6,7)$. Intrinsic brain networks are characterized by coherent ongoing activity at slow frequency $(<0.1 \mathrm{~Hz})(8)$ and have been demonstrated to be candidates for such network-based pathology spread $(4,5,7,9,10)$. Specifically, NDH suggests that each neurodegenerative disease may start in neuronal populations of preferentially targeted intrinsic network and progressively spread to connected regions within and then outside the network $(4,5,9)$. Proteins, which are believed to be responsible for disease pathogenesis such as amyloid- $\beta$ and tau in $\mathrm{AD}$, are known to disturb synaptic activity and axonal transport, which lead to a reduction in brain network integrity (11-14). In particular, it has been demonstrated that tau proteins may be able to travel across neurons, suggesting the expansion of pathology along connectivity pathways $(12,15)$.

Modern neuroimaging facilitates multimodal in vivo characterization of brain changes in neurodegenerative syndromes (16). For 
example, ${ }^{18} \mathrm{~F}$-FDG PET and structural MRI, respectively, detect regional hypometabolism, which reflects lowered regional neural activity, and atrophy, which reflects neurodegeneration, and both procedures are used for individual diagnostics (17-19). Restingstate functional MRI (rs-fMRI) facilitates the measurement of coherent ongoing brain activity, which reflects patterns of intrinsic functional connectivity including those of intrinsic brain networks $(8,20)$. Recently, on the basis of these advances in neuroimaging, several biomarkers have been proposed to differentiate between various neurodegenerative syndromes in individual patients (18,19,21-27). However, neuroimaging-based distinction between neurodegenerative syndromes is a challenge, because valid largescale models, which are both available for different syndromes and informative for macroscopic imaging, are rare (28).

The main question of this study is whether multimodal neuroimaging guided by the $\mathrm{NDH}$ can separate individual patients with different neurodegenerative syndromes. To obtain initial evidence, we performed a preliminary study, which combines multimodal imaging on a hybrid PET/MR scanner following concepts of the NDH and applying canonical multivariate pattern classification. Specifically, we focused on patients with $\mathrm{AD}$ and subtypes of FTLD, namely behavioral variant frontotemporal dementia (bvFTD), semantic dementia (SD), and progressive nonfluent aphasia (PNFA), and correspondingly core regions of 4 intrinsic networks, which have been previously linked with these 4 syndromes based on the NDH (4). Patients were assessed by rs-fMRI, ${ }^{18} \mathrm{~F}$-FDG PET, and structural MRI within 1 imaging session. We measured multimodal properties of network cores including intrinsic functional connectivity, regional metabolism, and gray matter volume. Concretely, for each patient and for each network core, we calculated degree centrality (DC) as surrogate for intrinsic functional connectivity, regional ${ }^{18}$ F-FDG-glucose metabolism (MET) as surrogate for regional activity, and voxel-based morphometry (VBM) as surrogate for regional gray matter volume. We hypothesized that such multimodal properties separate individual patients with different neurodegenerative syndromes. To test this hypothesis, support vector machine (SVM) was applied to patterns of outcome measures to estimate classification accuracy of individual patients.

\section{MATERIALS AND METHODS}

\section{Patients}

Forty patients with dementia due to different neurodegenerative syndromes (20 AD, 11 bvFTD, 4 SD, 5 PNFA) were included in this hybrid PET/MR study. A summary of subjects' demographics and relevant clinical information is listed in Table 1 and the supplemental data (supplemental materials are available at http://jnm.snmjournals.org).

\section{Data Acquisition and Preprocessing}

This study was registered and approved by the medical ethical board of the Technische Universität München in line with Human Research Committee guidelines of the Technische Universität München. Scanning of patients on the hybrid PET/MR scanner and subsequent imaging data preprocessing followed standard protocols of our center and have been described previously (details are provided in the supplemental data) (29-31).

\section{Definition of Intrinsic Network Cores}

On the basis of the NDH, AD, bvFTD, SD, and PNFA are associated with distinct intrinsic brain networks, which are preferentially affected in each disorder. It has been demonstrated that core regions of these networks overlap strikingly with peak atrophy in patients affected by the respective syndromes (4). We selected 4 core regions of each network, respectively, according to Seeley et al.: for $\mathrm{AD}$, right angular gyrus (RAng) of the default mode network with coordinates $(52,-58$, 36); for bvFTD, right frontoinsular cortex of the salience network with $(35,24,5)$; for SD, left temporal lobe of a temporal pole-anterior cingulate centered network with $(-44,14,-25)$; and for PNFA, left inferior frontal gyrus of left lateralized fronto-parietal language network with $(-43,15,27)$. We used MarsBaR (http://marsbar. sourceforge.net) to create 4 spheric regions of interest (ROIs with 10-mm radius) for each coordinate (Fig. 1). Subsequently, measures for regional functional connectivity, metabolism, and gray matter volume were extracted from these cores for each patient.

\section{Outcome Measures}

DC as Surrogate for Functional Connectivity. DC of functional connectivity of a given region reflects the sum of all functional connectivity weights, which are connected to the region (32). We performed calculation of voxelwise DC by canonical procedures as implemented in the REST toolkit (http://www.restfmri.net) (33). Specifically, for each subject, a whole-brain DC map was obtained by calculating for each voxel $i$ the sum of Fisher r-to-z normalized Pearson correlation coefficients $z_{i j}$ for all other voxels $j$ of the brain. Subsequently, the voxelwise DC values and the averaged DC values were extracted from each of the 4 core regions (RAng, right frontoinsular cortex, left temporal lobe, left inferior frontal gyrus) for each subject.

MET as Surrogate for Regional Metabolism. Preprocessed ${ }^{18} \mathrm{~F}-\mathrm{FDG}$ PET images were scaled by normalization of whole-brain ${ }^{18} \mathrm{~F}$-FDG uptake values to cerebellar vermis ${ }^{18} \mathrm{~F}$-FDG uptake $(34,35)$ and were spatially smoothed using a gaussian kernel full width at half maximum of $12 \mathrm{~mm}$. Afterward, from 4 core ROIs, we extracted voxelwise MET values from the normalized ${ }^{18} \mathrm{~F}-\mathrm{FDG}$ map of each subject. To be consistent with DC, we extracted the voxelwise and the averaged ${ }^{18} \mathrm{~F}$-FDG uptake values.

VBM as Surrogate for Gray Matter Volumes. To detect gray matter volume alterations within the 4 network cores, we followed a VBM protocol described in a previous study $(29,36)$. Briefly, we used the VBM8 toolbox (http://dbm.neuro.uni-jena.de/vbm.html) to analyze gray matter volume. T1-weighted images were corrected for bias-field inhomogeneity; registered using linear (12-parameter affine) and nonlinear transformations; and tissue-classified into gray matter, white matter, and cerebrospinal fluid within the same generative model. Then, the resulting gray matter images were modulated to account for volume changes that result from the normalization process. We considered nonlinear volume changes to control head size differences. Subsequently, our images were smoothed with a gaussian kernel of $8 \mathrm{~mm}$ (full width at half maximum). To be consistent with DC and MET, we extracted the voxelwise and averaged VBM values from core ROIs.

Multimodal Outcome Measure. Voxelwise multimodal outcome measures were created by concatenating unimodal voxelwise measures, respectively, into corresponding vectors $<$ DC, MET, VBM $>_{\mathrm{vx}}$. Voxelwise multimodal outcome vectors were of main interest in the study, because they preserve maximal imaging information.

\section{Classification of Individual Patients}

SVM. Canonical SVM was used to classify individual patients based on their multimodal voxelwise outcome measures. The basic idea of SVM procedures is to construct a separating hyperplane between the training instances (outcome measures of interest) of 2 classes (i.e., 2 groups of patients such as patients with AD vs. others) (details are provided in the supplemental data) (37).

Validation of Classification. Leave-one-out cross-validation was applied to validate SVM-based classification - that is, for each round of training, 1 subject was removed from the 2 classes and used for testing whereas the remaining data were used for training (resulting in 40 rounds of validation). Across rounds of validation, classification accuracy is then the percentage of cases that were assigned correctly to the clinical diagnosis $(18,19,38)$. The validity of classification was evaluated by the following measures: accuracy, sensitivity, and specificity. 
TABLE 1

Demographic and Clinical Data of Patients

\begin{tabular}{|c|c|c|c|c|c|}
\hline Characteristic & $\mathrm{AD}(n=20)$ & bvFTD $(n=11)$ & $\mathrm{SD}(n=4)$ & PNFA $(n=5)$ & $P$ \\
\hline Age & $72.2(8.7)$ & $61.0(9.6)$ & $65.7(6.0)$ & $68.0(7.9)$ & $0.010^{*}$ \\
\hline Sex (male) & 13 & 9 & 2 & 1 & 0.119 \\
\hline Duration of symptom (y) & $4.92(1.9)$ & $6.91(4.5)$ & $5.50(3.7)$ & $3.60(1.5)$ & 0.29 \\
\hline Mini-mental state examination & $22.03(4.61)$ & $23.73(7.0)$ & $18.75(12.8)$ & 19.00 (5.92) & 0.062 \\
\hline
\end{tabular}

${ }^{*} P$ value indicates the statistically significant difference between 4 groups using ANOVA.

Group comparisons by ANOVA, except for sex by Kruskal-Wallis test.

$\mathrm{AD}=$ Alzheimer disease; bvFTD = behavioral variant frontotemporal dementia; $\mathrm{SD}=$ semantic dementia; PNFA = progressive nonfluent aphasia.

\section{Control Analyses}

Nonvoxelwise Multimodal Values. To control for the impact of multimodality and voxelwise account, correspondent averaged or unimodal outcome vectors were defined. Specifically, to control findings for information loss due to averaging, $<\mathrm{DC}, \mathrm{MET}, \mathrm{VBM}>$ av was defined and used for SVM-based classification. In addition, further correspondent unimodal voxelwise/averaged outcome vectors (e.g., $<\mathrm{DC}\rangle_{\mathrm{vx}}$ or $\langle\mathrm{DC}\rangle{ }_{\text {av }}$ ) were used to control information loss due to ignoring multimodal aspects of neurodegeneration-induced brain changes.

Classification Analysis Based on Core Region Not Derived from $N D H$. To evaluate group-separating power of NDH-based regions versus non-NDH-based regions, we extracted the voxelwise unimodal and multimodal values from another ROI not derived from the NDH (i.e., the right primary motor cortex with coordinates $(12,-16,74)$ (39), then we applied SVM to compare the classification results from this ROI with the above-mentioned core regions.

Statistical Comparison Between Multimodal Voxelwise and Nonmultimodal/Voxelwise Values. To evaluate group-separating power of multimodal voxelwise versus other approaches, we used the McNemar test to calculate the statistical significance between voxelwise multimodal $<$ DC, MET, VBM $>_{\text {vx }}$ values and other values (such as unimodal averaged $\langle\text { DC }\rangle_{\text {vx }}$ or $<$ MET $\rangle_{\text {vx }}$ or $<$ VBM $\rangle_{\text {vx }}$ ) $(40)$.

\section{RESULTS}

For each neurodegenerative disease and corresponding network core, SVM together with leave-one-out cross-validation was applied to voxelwise multimodal outcome measures $<$ DC, MET, $\mathrm{VBM}>{ }_{\mathrm{vx}}$ to separate patients of the given disease from those of other syndromes (Table 2). Classification accuracies were $77.5 \%$ for $\mathrm{AD}$ versus others, $82.5 \%$ for bvFTD versus others, $97.5 \%$ for SD versus others, and $87.5 \%$ for PNFA versus others.

To evaluate the use of multimodal versus unimodal voxelwise outcome measures, we studied classification results based on unimodal voxelwise $\langle\mathrm{DC}\rangle_{\mathrm{vx}},\langle\mathrm{MET}\rangle_{\mathrm{vx}}$, and $\langle\mathrm{VBM}\rangle_{\mathrm{vx}}$ (Table 2). Results were as follows: for $<\mathrm{DC}\rangle_{\mathrm{vx}}, 70 \%$ for $\mathrm{AD}$ versus others, $70 \%$ for bvFTD versus others, $77.5 \%$ for SD versus others, and $87.5 \%$ for PNFA versus others; for $<$ MET $>_{v x}, 72.5 \%$ for AD versus others, $60 \%$ for bvFTD versus others, $97.5 \%$ for SD versus others, and $85 \%$ for PNFA versus others; and for $<\mathrm{VBM}\rangle_{\mathrm{vx}}$, $32.5 \%$ for AD versus others, $72.5 \%$ for bvFTD versus others, $92.5 \%$ for SD versus others, and $82.5 \%$ for PNFA versus others. Statistical comparisons revealed that the voxelwise multimodal classification results were superior to unimodal approaches in separating individual patients. In particular, voxelwise multimodal $<$ DC, MET, $\mathrm{VBM}>_{\mathrm{vx}}$ accuracies were significantly different compared with
$<\mathrm{DC}\rangle_{\mathrm{vx}}$ or $<$ MET $\rangle_{\mathrm{vx}}$ or $\left.<\mathrm{VBM}\right\rangle_{\mathrm{vx}}$ for both $\mathrm{AD}$ and bvFTD groups, compared with $\langle\mathrm{DC}\rangle_{\mathrm{vx}}$ or $\langle\mathrm{VBM}\rangle_{\mathrm{vx}}$ for the SD group, and compared with $<$ MET $>_{{ }_{\mathrm{vx}}}$ for the PNFA group $(P<0.05)$ (Table 2$)$.

To evaluate the use of averaged versus voxelwise multimodal outcome measures, we assessed classification results based on averaged multimodal $<$ DC, MET, VBM $>$ av. The accuracy results based on the averaged values were $72.5 \%$ for $\mathrm{AD}$ versus others, $72.5 \%$ for bvFTD versus others, $90 \%$ for SD versus others, and $87.5 \%$ for PNFA versus others, which were significantly lower than accuracies for corresponding voxelwise values $(P<0.05)$, except for PNFA. In PNFA, the averaged approach demonstrated already high classification accuracy. These results showed that averaged multimodal outcomes performed worse than voxelwise measures in separating individual patients.

To evaluate the impact of NDH-based versus non-NDH-based ROIs for separating individual patients, the group-separating power of a ROI outside NDH-based networks of interest was investigated. Classification results based on the voxelwise multimodal $<\mathrm{DC}$,

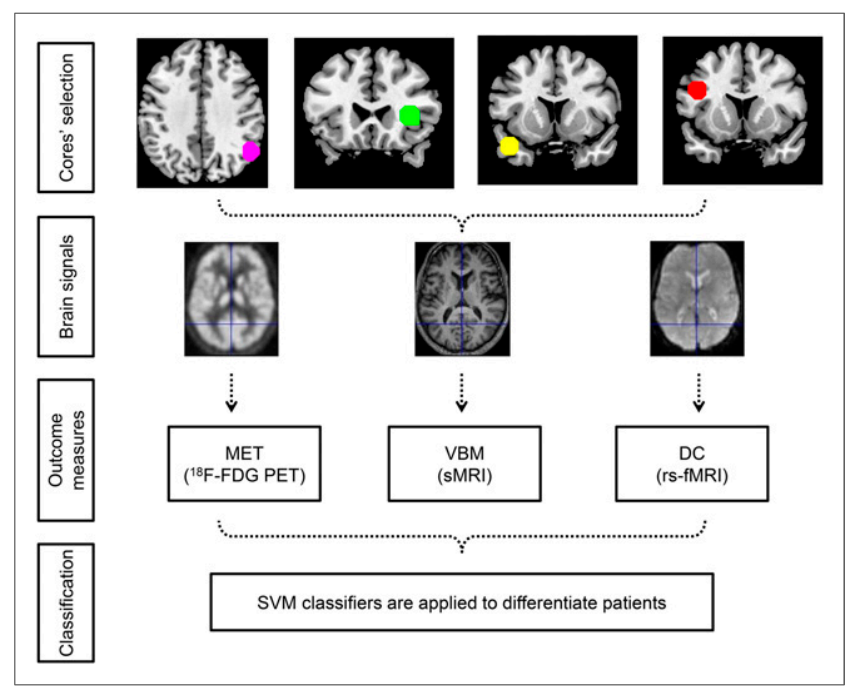

FIGURE 1. Study flowchart. Main core region of each network was created based on previous study (4). Subsequently, voxelwise values and averaged values of 3 modalities were extracted from each core for all subjects. Then, SVM was applied to classify each patient. DC = degree centrality; MET = glucose metabolism; rs-fMRI = resting-state functional magnetic resonance imaging; sMRI = structural magnetic resonance imaging; SVM = support vector machine; VBM = voxel-based morphometry. 
TABLE 2

Classification Based on Voxelwise Unimodal and Multimodal Outcome Measures

\begin{tabular}{|c|c|c|c|c|c|}
\hline Measurement & Validity & $\begin{array}{l}\text { AD vs. others } \\
\text { (AD core) }\end{array}$ & $\begin{array}{l}\text { bvFTD vs. others } \\
\text { (bvFTD core) }\end{array}$ & $\begin{array}{l}\text { SD vs. others } \\
\text { (SD core) }\end{array}$ & $\begin{array}{l}\text { PNFA vs. others } \\
\text { (PNFA core) }\end{array}$ \\
\hline \multirow[t]{4}{*}{$<\mathrm{DC}>_{\mathrm{vx}}$} & Accuracy (\%) & 70 & 70 & 77.5 & 87.5 \\
\hline & Sensitivity (\%) & 55 & 36.3 & 100 & 0 \\
\hline & Specificity (\%) & 85 & 82.7 & 75 & 100 \\
\hline & Uni vs. multi & $P<0.001^{*}$ & $P<0.001^{*}$ & $P=0.003^{\star}$ & $P=1.0$ \\
\hline \multirow[t]{4}{*}{$<$ MET $>_{\text {vx }}$} & Accuracy (\%) & 72.5 & 60 & 97.5 & 85 \\
\hline & Sensitivity (\%) & 70 & 36.3 & 100 & 40 \\
\hline & Specificity (\%) & 75 & 68.9 & 97.2 & 91.2 \\
\hline & Uni vs. multi & $P<0.001^{\star}$ & $P<0.001^{\star}$ & $P=1.0$ & $P<0.001^{*}$ \\
\hline \multirow[t]{4}{*}{$<\mathrm{VBM}>_{\mathrm{vx}}$} & Accuracy (\%) & 32.5 & 72.5 & 92.5 & 82.5 \\
\hline & Sensitivity (\%) & 35 & 45.4 & 50 & 0 \\
\hline & Specificity (\%) & 30 & 82.7 & 97.5 & 94.2 \\
\hline & Uni vs. multi & $P<0.001^{\star}$ & $P<0.001^{\star}$ & $P<0.001^{*}$ & $P=0.25$ \\
\hline \multirow[t]{3}{*}{$<\mathrm{DC}, \mathrm{MET}, \mathrm{VBM}>_{\mathrm{vx}}$} & Accuracy (\%) & 77.5 & 82.5 & 97.5 & 87.5 \\
\hline & Sensitivity (\%) & 80 & 54.5 & 100 & 0 \\
\hline & Specificity (\%) & 75 & 93.1 & 97.2 & 100 \\
\hline
\end{tabular}

Uni vs. multi and corresponding $P$ value indicate statistically significant difference between unimodal $\left(<\mathrm{DC}>{ }_{\mathrm{vx}}\right.$ or $<$ MET $>{ }_{\mathrm{vx}}$ or $<$ VBM $>_{v x}$ ) vs. multimodal $<$ DC, MET, VBM $>_{v x}$ values.

$A D=$ Alzheimer disease; bvFTD = behavioral variant frontotemporal dementia; $S D=$ semantic dementia; PNFA = progressive nonfluent aphasia; $\mathrm{DC}$ = degree centrality as proxy for regional functional connectivity; vx = voxel-wise; MET = glucose metabolism as proxy for regional activity; VBM = voxel-based morphometry as proxy for regional gray matter volume.

MET, $\mathrm{VBM}>_{\mathrm{vx}}$ values from a ROI in the primary motor cortex were $45 \%$ for $\mathrm{AD}$ versus others, $60 \%$ for bvFTD versus others, $90 \%$ for SD versus others, and $87.5 \%$ for PNFA versus others, which were significantly lower $(P<0.001)$ than accuracies for corresponding NDH-based ROIs, except of PNFA.

\section{DISCUSSION}

To test whether multimodal imaging based on the brain NDH might be useful to separate individual patients with different neurodegenerative syndromes, we assessed patients with AD, bvFTD, SD, and PNFA in a hybrid PET/MR scanner. We defined the multimodal voxelwise outcome measure $<\mathrm{DC}, \mathrm{MET}, \mathrm{VBM}>_{\mathrm{vx}}$, which reflects regional degree centrality of functional connectivity, metabolism of regional activity, and voxel-based morphometry of gray matter volume. On the basis of core regions of 4 intrinsic brain networks, which are specific for patients' syndromes, $<$ DC, MET, VBM $>_{\text {vx }}$ classified individual patients accurately for their diagnostic category. These preliminary results demonstrate that combining advanced multimodal neuroimaging with distinct network-based degeneration patterns separates individual patients of different neurodegenerative syndromes.

We found that $<$ DC, MET, VBM $>_{\text {vx }}$ separated individual patients with $\mathrm{AD}$, bvFTD, SD, or PNFA, respectively, with classification accuracy ranging from $77.5 \%$ to $97.5 \%$ (Table 2). $<$ DC, MET, VBM $>_{v x}$ was defined as a concatenated vector based on 3 unimodal outcome measures (e.g., $<\mathrm{DC}>{ }_{\mathrm{vx}}$ ) (Fig. 1). Unimodal outcome measures, in turn, were derived from standard procedures of unimodal imaging data analysis. Critically, $<$ DC, MET, VBM $>_{v x}$ was restricted to regions, which were derived from disease-specific intrinsic networks derived from the NDH $(4,6)$. To be independent from the current sample and to avoid circular analysis, exact coordinates of core regions were derived from a previous study (4). Recent findings provided overwhelming evidence that distinct intrinsic brain networks are primarily affected by different neurodegenerative syndromes $(4,5,7)$. More specifically, several findings suggest that critical proteins of neurodegeneration such as tau spread in a prionlike transsynaptic way along brain networks $(12,13,15)$. Our finding of network core-based classification of individual patients with different neurodegenerative syndromes supports such an NDH; but beyond regional information, one should note that used imaging-based information of these cores is not specific to any of the neurodegenerative disorders, especially those with similar underlying pathologies.

\section{Classification Accuracies}

Classification accuracies for different syndromes range from $77.5 \%$ to $97.5 \%$ (Table 2). These accuracies are comparable with or superior to those of similar studies. For example, a previous study applied VBM and diffusion tensor imaging (DTI) to separate FTLD subtypes (i.e., bvFTD, SD, and PNFA) from healthy controls. The authors revealed that DTI measurement, particularly radial diffusivity, provided better accuracies $(67.6 \%-81.4 \%)$ than gray matter atrophy $(45.7 \%-65.7 \%)$ or white matter atrophy $(47.4 \%-59.2 \%)(41)$. Moreover, Dukart et al. demonstrated classification accuracy of $60.0 \%$ using structural MRI, $80.0 \%$ using ${ }^{18}$ F-FDG PET, and $94.3 \%$ using combined ${ }^{18} \mathrm{~F}$-FDG PET and structural MRI to separate AD from FTLD patients (18). This study supports our result that multimodal imaging is superior to classify individual patients with different neurodegenerative syndromes. 
However, a recent study demonstrated that the combination of rs-fMRI, DTI, and anatomic MRI did not significantly improve classification accuracy compared with the unimodal measurements (27). Discrepancy between our results and these results might be due to different imaging modalities and their whole-brain versus our approach derived from the NDH. In addition, Tang et al. applied an automated image-based classification procedure to separate patients with different neurodegenerative disorders using ${ }^{18}$ F-FDG PET and spatial covariance analysis and achieved $84 \%$ sensitivity and $97 \%$ specificity for idiopathic Parkinson disease, $88 \%$ sensitivity and $94 \%$ specificity for progressive supranuclear palsy, and $85 \%$ sensitivity and $96 \%$ specificity for multiple system atrophy. This approach worked well with unimodal imaging and does not require a priori knowledge of cores from the NDH (42).

\section{Multimodal Versus Unimodal Outcome Measures}

In general, classification accuracies based on multimodal outcomes $<$ DC, MET, VBM $>_{\mathrm{vx}}$ were higher than those based on unimodal measures such as $\langle\mathrm{DC}\rangle_{\mathrm{vx}}$ (Table 2). Unimodal values separated syndromes with different success. For example, $\langle\mathrm{DC}\rangle_{\mathrm{vx}}$ separated each disease with more than $70 \%$ accuracy, whereas $<$ MET $\rangle_{\mathrm{vx}}$ separated $\mathrm{AD}, \mathrm{SD}$, and PNFA with more than $70 \%$ accuracy but not bvFTD. The results were not robust for $\langle\mathrm{VBM}\rangle_{\mathrm{vx}}$, probably because our patients were in the mild to moderate stage of diseases. Together with successful multimodal disease classification, this means that although unimodal outcomes are partly different across syndromes, multimodal values boost this difference. This finding suggests that pathologic changes of specific syndromes are differentially reflected by different aspects of changes (i.e., changes in regional functional connectivity, activity, and brain structure), which are detected by different views into the brain. Therefore, our finding suggests that multimodal and regionally specific imaging markers have a higher potential to serve as successful biomarkers for neurodegenerative syndromes. For PNFA versus others, $\langle\mathrm{DC}\rangle\rangle_{\text {vx }}$ accuracy equals that of the multimodal approach $(87.5 \%)$. Similarly, for SD versus others $<$ MET $>_{\text {vx }}$ accuracy equals that of the multimodal approach $(97.5 \%)$. These results may not be generalizable because of the small sample size of these groups, but further studies with larger samples should focus on this issue-that is, for specific disorders such as PNFA and SD, a specific imaging modality may provide accuracy comparable to that of the multimodal approach.

\section{Voxelwise Versus Averaged Outcome Measures}

Results indicate further that a voxelwise outcome measure has a better performance for classification than regional averaged measures. This finding suggests that within core regions, gradients of brain measures provide valuable information to separate syndromes. This point highlights the value of voxelwise outcome measures, which are often ignored when using averaging procedures.

\section{Classification on Core Region Not Derived from the NDH}

The classification results based on voxelwise multimodal $<\mathrm{DC}$, MET, VBM $>{ }_{\mathrm{vx}}$ from the motor core were worse than the 4 cores derived from the NDH, particularly in $\mathrm{AD}$, bvFTD, and SD groups. These findings support our hypothesis based on the NDH and highlight the role of network abnormalities in pathophysiology of neurodegenerative diseases.

\section{Concatenated Multimodal Outcome Measures Versus Integrated Measures}

We used simple concatenation to build multimodal outcome measures. Concatenation preserves voxelwise and multimodal information but does not integrate measures to reflect physiologically relevant relationships across values. For example, one might suggest that aberrant DC is systematically linked with aberrant local metabolism (30), and integrative values might reflect such a link (e.g., spatial correlation between DC and MET) and can be used for classification (9). Future research is necessary to develop integrated measures of different aspects of activity, which are pathophysiologically relevant and valid for disease classification.

\section{Limitations and Strengths}

The current study has several limitations. First, the sample size of this study is small particularly in the FTLD subgroups, limiting the power of our findings. However, the prevalence of FTLD patients particularly of SD and PNFA is low so that it is difficult to recruit a large patient sample in a monocentric study. Second, our findings are not validated for several aspects, which range from diagnostic validation to methodologic-analytic issues. For example, the validity of results depends critically on the validity of the diagnosis of neurodegenerative syndromes (43), which should be optimally based on neuropathologic findings. Although leave-oneout cross-validation of classification results was performed, validation of findings in an independent sample of patients might be helpful. Third, classification results might be biased, because clinical diagnostic procedures involved qualitative inspection of ${ }^{18} \mathrm{~F}$ FDG PET and structural MRI data. Fourth, as mentioned above, our multimodal outcome measure is based on regional neuroimaging properties and it is not based on direct pathophysiologic aspects specific for each neurodegenerative syndrome. Fifth, application of this regional approach based on the NDH is not feasible for other neurodegenerative variants when prior knowledge of specific cores is unavailable. With these points taken together, our study fulfills criteria of a preliminary study, which searches for initial evidence that combining the NDH and multimodal imaging is helpful for individual patient separation in different neurodegenerative syndromes. On the other hand, our study has some strengths: first, the idea of combining the NDH and multimodal imaging is simple. Second, the NDH, which has been strongly confirmed by many different studies, is applicable for different neurodegenerative syndromes. Third, to our best knowledge, this is the first study on FTLD patients using the simultaneous PET/MR measurement. Finally, applied analytic methods are well elaborated and widely used and available. Given the high intraindividual variability of neural functions, the simultaneous PET and MRI measurement in a single examination provides unique opportunities to study the relationship between different parameters in the same condition (29-31). However, separate MRI and PET imaging could be used also to assess metabolism, atrophy, and connectivity in neurodegenerative syndromes successfully. In addition, ${ }^{18}$ F-FDG PET imaging may eventually be substituted by blood flow marker such as arterial spin labeling $(44,45)$, which would allow this technique to be applied to MRI-based studies when PET imaging is not available.

\section{CONCLUSION}

We provide preliminary evidence that applying multimodal imaging based on assumptions derived from the network neurodegeneration hypothesis has the potential to yield imaging biomarkers for neurodegenerative syndromes.

\section{DISCLOSURE}

The costs of publication of this article were defrayed in part by the payment of page charges. Therefore, and solely to indicate this 
fact, this article is hereby marked "advertisement" in accordance with 18 USC section 1734. This work was supported by the German Federal Ministry of Education and Research (BMBF 01ER0803), German research foundation (DFG) grants (Nr. DR 445/4-1, DR 445/5-2, and SFB 824), the Kommission für Klinische Forschung of the Klinikum Rechts der Isar der Technischen Universität München (KKF 8765162), the BMBF (Federal Ministry of Education and Research, Germany): Competence Net Neurodegenerative Dementias (project: FTLD Consortium), the German Academic Exchange service (DAAD), the National Natural Science Foundation of China $(61403062,61433014)$, and the China Postdoctoral Science Foundation (2015M580786). No other potential conflict of interest relevant to this article was reported.

\section{ACKNOWLEDGMENTS}

We thank all patients and their relatives for their participation in the study. Furthermore, we thank the staff of the Departments of Psychiatry and Psychotherapy and also Nuclear Medicine for their help in recruitment and data collection.

\section{REFERENCES}

1. Bishop NA, Lu T, Yankner BA. Neural mechanisms of ageing and cognitive decline. Nature. 2010;464:529-535.

2. Sorg C, Riedl V, Muhlau M, et al. Selective changes of resting-state networks in individuals at risk for Alzheimer's disease. Proc Natl Acad Sci USA. 2007;104:1876018765.

3. Greicius MD, Srivastava G, Reiss AL, Menon V. Default-mode network activity distinguishes Alzheimer's disease from healthy aging: evidence from functional MRI. Proc Natl Acad Sci USA. 2004;101:4637-4642.

4. Seeley WW, Crawford RK, Zhou J, Miller BL, Greicius MD. Neurodegenerative diseases target large-scale human brain networks. Neuron. 2009;62:42-52.

5. Zhou J, Greicius MD, Gennatas ED, et al. Divergent network connectivity changes in behavioural variant frontotemporal dementia and Alzheimer's disease. Brain. 2010;133:1352-1367.

6. Palop JJ, Chin J, Mucke L. A network dysfunction perspective on neurodegenerative diseases. Nature. 2006;443:768-773.

7. Greicius MD, Kimmel DL. Neuroimaging insights into network-based neurodegeneration. Curr Opin Neurol. 2012;25:727-734.

8. Fox MD, Raichle ME. Spontaneous fluctuations in brain activity observed with functional magnetic resonance imaging. Nat Rev Neurosci. 2007;8:700-711.

9. Myers N, Pasquini L, Gottler J, et al. Within-patient correspondence of amyloid-beta and intrinsic network connectivity in Alzheimer's disease. Brain. 2014;137:2052-2064.

10. Tahmasian M, Bettray LM, van Eimeren T, et al. A systematic review on the applications of resting-state fMRI in Parkinson's disease: does dopamine replacement therapy play a role? Cortex. 2015;73:80-105.

11. Palop JJ, Mucke L. Amyloid-beta-induced neuronal dysfunction in Alzheimer's disease: from synapses toward neural networks. Nat Neurosci. 2010;13:812-818.

12. Raj A, Kuceyeski A, Weiner M. A network diffusion model of disease progression in dementia. Neuron. 2012;73:1204-1215.

13. Frost B, Diamond MI. Prion-like mechanisms in neurodegenerative diseases. Nat Rev Neurosci. 2010;11:155-159.

14. Braak H, Del Tredici K. Alzheimer's pathogenesis: is there neuron-to-neuron propagation? Acta Neuropathol (Berl). 2011;121:589-595.

15. de Calignon A, Polydoro M, Suarez-Calvet M, et al. Propagation of tau pathology in a model of early Alzheimer's disease. Neuron. 2012;73:685-697.

16. Pievani M, de Haan W, Wu T, Seeley WW, Frisoni GB. Functional network disruption in the degenerative dementias. Lancet Neurol. 2011;10:829-843.

17. Yun HJ, Kwak K, Lee JM. Alzheimer's disease neuroimaging I. Multimodal discrimination of Alzheimer's disease based on regional cortical atrophy and hypometabolism. PLoS One. 2015;10:e129250.

18. Dukart J, Mueller K, Horstmann A, et al. Combined evaluation of FDG-PET and MRI improves detection and differentiation of dementia. PLoS One. 2011;6:e18111.
19. Dukart J, Mueller K, Barthel H, et al. Meta-analysis based SVM classification enables accurate detection of Alzheimer's disease across different clinical centers using FDG-PET and MRI. Psychiatry Res. 2013;212:230-236.

20. Fox MD, Snyder AZ, Vincent JL, Corbetta M, Van Essen DC, Raichle ME. The human brain is intrinsically organized into dynamic, anticorrelated functional networks. Proc Natl Acad Sci USA. 2005;102:9673-9678.

21. Schroeter ML, Neumann J. Combined imaging markers dissociate Alzheimer's disease and frontotemporal lobar degeneration: an ALE meta-analysis. Front Aging Neurosci. 2011;3:10.

22. Diehl-Schmid J, Grimmer T, Drzezga A, et al. Decline of cerebral glucose metabolism in frontotemporal dementia: a longitudinal ${ }^{18} \mathrm{~F}-\mathrm{FDG}-\mathrm{PET}$-study. Neurobiol Aging. 2007;28:42-50.

23. Diehl J, Grimmer T, Drzezga A, Riemenschneider M, Forstl H, Kurz A. Cerebral metabolic patterns at early stages of frontotemporal dementia and semantic dementia: a PET study. Neurobiol Aging. 2004;25:1051-1056.

24. Rosen HJ, Gorno-Tempini ML, Goldman WP, et al. Patterns of brain atrophy in frontotemporal dementia and semantic dementia. Neurology. 2002;58:198-208.

25. Abdi H, Williams LJ, Beaton D, et al. Analysis of regional cerebral blood flow data to discriminate among Alzheimer's disease, frontotemporal dementia, and elderly controls: a multi-block barycentric discriminant analysis (MUBADA) methodology. J Alzheimers Dis. 2012;31:S189-S201.

26. Teipel SJ, Kurth J, Krause B, Grothe MJ. Alzheimer's disease neuroimaging I: the relative importance of imaging markers for the prediction of Alzheimer's disease dementia in mild cognitive impairment-beyond classical regression. Neuroimage Clin. 2015;8:583-593.

27. Dyrba M, Grothe M, Kirste T, Teipel SJ. Multimodal analysis of functional and structural disconnection in Alzheimer's disease using multiple kernel SVM. Hum Brain Mapp. 2015;36:2118-2131.

28. Pievani M, Filippini N, van den Heuvel MP, Cappa SF, Frisoni GB. Brain connectivity in neurodegenerative diseases: from phenotype to proteinopathy. Nat Rev Neurol. 2014;10:620-633.

29. Tahmasian M, Pasquini L, Scherr M, et al. The lower hippocampus global connectivity, the higher its local metabolism in Alzheimer disease. Neurology. 2015;84:1956-1963.

30. Riedl V, Bienkowska K, Strobel C, et al. Local activity determines functional connectivity in the resting human brain: a simultaneous FDG-PET/fMRI study. J Neurosci. 2014;34:6260-6266.

31. Tahmasian M, Eggers C, Riedl V, Sorg C, Drzezga A. Utilization of hybrid PET/ MR in neuroimaging. Basic Clin Neurosci. 2015;6:143-146.

32. Rubinov M, Sporns O. Complex network measures of brain connectivity: uses and interpretations. Neuroimage. 2010;52:1059-1069.

33. Song XW, Dong ZY, Long XY, et al. REST: a toolkit for resting-state functional magnetic resonance imaging data processing. PLoS One. 2011;6:e25031.

34. Chételat G, Desgranges B, Landeau B, et al. Direct voxel-based comparison between grey matter hypometabolism and atrophy in Alzheimer's disease. Brain. 2008;131:60-71.

35. Mevel K, Desgranges B, Baron JC, et al. Detecting hippocampal hypometabolism in mild cognitive impairment using automatic voxel-based approaches. Neuroimage. 2007;37:18-25.

36. Ashburner J, Friston KJ. Unified segmentation. Neuroimage. 2005;26:839-851.

37. Cortes C, Vapnik V. Support-vector networks. Mach Learn. 1995;20:273-297.

38. Shao J, Myers N, Yang Q, et al. Prediction of Alzheimer's disease using individual structural connectivity networks. Neurobiol Aging. 2012;33:2756-2765.

39. Anderson JS, Ferguson MA, Lopez-Larson M, Yurgelun-Todd D. Reproducibility of single-subject functional connectivity measurements. AJNR. 2011;32:548-555.

40. Japkowicz N, Shah M. Evaluating Learning Algorithms: A Classification Perspective. New York, NY: Cambridge University Press; 2011.

41. Zhang Y, Tartaglia MC, Schuff N, et al. MRI signatures of brain macrostructural atrophy and microstructural degradation in frontotemporal lobar degeneration subtypes. J Alzheimers Dis. 2013;33:431-444.

42. Tang CC, Poston KL, Eckert T, et al. Differential diagnosis of parkinsonism: a metabolic imaging study using pattern analysis. Lancet Neurol. 2010;9:149-158.

43. Klöppel S, Stonnington CM, Barnes J, et al. Accuracy of dementia diagnosis: a direct comparison between radiologists and a computerized method. Brain. 2008;131:2969-2974.

44. Chen Y, Wolk DA, Eddin JSR, et al. Voxel-level comparison of arterial spin-labeled perfusion MRI and FDG-PET in Alzheimer disease. Neurology. 2011;77:1977-1985.

45. Musiek ES, Chen YF, Korczykowski M, et al. Direct comparison of fluorodeoxyglucose positron emission tomography and arterial spin labeling magnetic resonance imaging in Alzheimer's disease. Alzheimers Dement. 2012;8:51-59. 